\title{
A Normal Bias Toward a Pictorially Defined Top in Line Bisection
}

\author{
Cory Toth and Andrew Kirk
}

\begin{abstract}
Background: We set out to determine whether separable visual and representational components underlie normal subjects' upward and distal biases in bisecting vertical and radial lines under visual guidance. Methods: Thirty-four normal subjects were asked to bisect lines oriented horizontally, vertically, and radially. Human silhouette figures were placed at either end of each line. These figures were presented upright or upside down in order to pictorially define a "top" to each line independent of the actual top of the visual field. Results: Although subjects erred toward the top of the visual field, they also demonstrated a significant bias toward the heads of the figures for lines in all spatial orientations. Conclusions: This result supports the existence of two biases: one toward the upper visual field, and another toward an internally represented "top" as suggested pictorially. These findings provide further support for the hypothesis that normal subjects' upward and distal biases on bisection of vertical and radial lines under visual guidance have both representational and visual-based components.
\end{abstract}

\begin{abstract}
RÉSUMÉ: Biais normal vers le haut dans l'hémisection d'une ligne. Introduction: Notre but était de déterminer, chez des sujets normaux, si des composantes représentationnelles et visuelles séparables sont en cause dans les biais vers le haut et à distance dans l'hémisection de lignes verticales et radiales sous guidage visuel. Méthodes: Nous avons demandé à trente-quatre sujets normaux de diviser en deux des lignes orientées de façon horizontale, verticale et radiale. Des silhouettes humaines étaient placées aux extrémités de chaque ligne. Ces figures étaient présentées en station debout ou tête en bas pour définir le haut de chaque ligne dans le plan du dessin indépendamment du haut du champ visuel. Résultats: Bien que les sujets faisaient erreur vers le haut du champ visuel, ils faisaient également preuve d'un important biais vers la tête des formes aux extrémités des lignes orientées dans toutes les directions. Conclusions: Ce résultat supporte l'existence de deux biais: l'un vers le haut du champ visuel et l'autre vers un haut représenté intérieurement tel que suggéré par le dessin. Ces observations appuient davantage l'hypothèse voulant que les biais vers le haut et à distance à l'hémisection de lignes verticales et radiales, sous guidage visuel chez le sujet normal, ont des composantes représentationnelles et visuelles.
\end{abstract}

Can. J. Neurol. Sci. 1996; 23: 110-113

Attention may be oriented along three axes in space: horizontal, vertical, and radial. Patients with neglect who misbisect lines presented along the vertical and radial axes have been reported by Shelton et al. ${ }^{1}$ and Mennemeier et al. ${ }^{2}$ Normal subjects also show a bias in bisection of non-horizontal lines. An upward bias has been reported in vertical line bisection as performed by normal subjects.' Radial lines are misbisected distal to the true midpoint when placed below eye level and are misbisected proximal to midpoint when placed above eye level. ${ }^{3}$

Shelton et al.' suggested that misbisection of radial lines distal to midpoint results from direction of attention away from the body under visual guidance, as vision is designed to detect far stimuli. However, Shelton et al. ' presented lines in a "radial-down" fashion, with the line below eye level. If a far peripersonal bias exists, then "radial-up" lines, that is radial lines presented above eye level, should also be misbisected distal to midpoint as well. However, Geldmacher and Heilman $^{3}$ found that radial lines presented above eye level are misbisected proximal to midpoint. Geldmacher and Heilman ${ }^{3}$ proposed a bias towards the top of the visual field which occurs as a result of retinotopic factors.

Jeerakathil and $\mathrm{Kirk}^{4}$ suggested that, in addition to a retinotopic bias, normal subjects may also have an attentional bias toward the top of an object based on an internal (non-visual apparatus-related) representation of top and bottom. This hypothesis suggests that normal subjects display a bias toward what they believe to be the top of the line itself. Jeerakathil and Kirk $^{4}$ tested this hypothesis by having normal subjects bisect lines with directional labels ("TOP" and "BOTTOM") at either end of each line. Normal subjects displayed a significant bias toward the label TOP for lines in most spatial orientations. ${ }^{4}$ Horizontal line bisection provided the strongest argument for an

From the Division of Neurology, Department of Medicine, University of Saskatchewan, Saskatoon.

RECEIVED AUGUST 15, 1995. ACCEPTED IN FINAL FORM OCTOBER 26, 1995. Reprint requests to: Dr. Andrew Kirk, Division of Neurology, Department of Medicine, Royal University Hospital, 103 Hospital Drive, Saskatoon, Saskatchewan, Canada S7N 0W8 
internal representation - the bisection error actually changed direction depending on which end of the line was labelled TOP. Jeerakathil and $\mathrm{Kirk}^{4}$ proposed two components in misbisection of vertical and radial-down lines: 1) a bias towards the top as determined by the visual field and 2) a bias towards the internal representation of an object's top. They discounted the importance of a radial or peripersonal visual bias and suggested that the "radial" bias may actually be a vertical bias based on a visual or representational frame of reference. More recently, Kirk and Boyle ${ }^{5}$ demonstrated that peripersonal visual neglect in a stroke patient could be better explained as vertical neglect based upon a visual or representational frame of reference.

Jeerakathil and $\mathrm{Kirk}^{4}$ chose the verbal labels TOP and BOTTOM as the most salient of all stimuli. However, a potential weakness of that study is the fact that the word TOP is shorter than the word BOTTOM which may lead to a "pyramid" appearance for the line, perhaps creating an optical illusion which might provide an unanticipated bias during bisection. Verbal cues also depend upon processing by left hemisphere language areas. A non-verbal cue may be processed elsewhere. Therefore, the use of a non-verbal cue to induce a representation of an object's top and bottom contrary to the top and bottom of the visual field could provide further support for Jeerakathil and Kirk' ${ }^{4}$ hypothesis, or it might instead demonstrate that their findings were artefactual.

The present study investigates the effects of non-verbal directional cues on line bisection. Black silhouette human figures were presented at each end of the lines to be bisected. The human figure was selected so as to provide an obvious top (head) and bottom (feet) to the line to be bisected. Unlike verbal labels, identical figures can be presented at each end of the line so that unintended biases based on a difference in word length can be avoided. Figures can be presented symmetrically and are universally recognizable. A bias towards the heads of the figures would support the existence of a bias toward subjects' representation of the top of an object.

\section{MeThods}

\section{Subjects}

Thirty-four healthy, right-handed subjects participated, 13 men and 21 women with a mean age of 36 years (S.D. 19). Right-handedness was determined by subject self-reporting. No subjects reported any history of previous head trauma, stroke, brain tumour, multiple sclerosis, or any other forms of neurologic illness. Informed consent was obtained from each subject once the procedure had been explained fully.

\section{Stimuli}

The lengths and widths of the black lines presented for bisection were identical to those used by Jeerakathil and Kirk. ${ }^{4}$ Lines were $2.5 \mathrm{~mm}$ wide and of six different lengths $(20,22,24,26$, 28 , and $30 \mathrm{~cm}$ ). Lines were centred on a 21.6 by $35.5 \mathrm{~cm}$ white page. Instead of verbal labels, silhouettes of a human figure, 6 $\mathrm{cm}$ from head to toe, were placed at both ends of each line as shown in Figure 1, with one figure on each side of the line, giving four figures in total. All figures had their midline parallel with the black line with all figures' heads pointing in the same direction. The medialmost portion of the figure was $5 \mathrm{~mm}$ from the lateral edge of the line. The figures extended $12 \mathrm{~mm}$ past the end of the line, allowing the tops and bottoms of the figures to be the same distance from the ends of the line as the letters used by Jeerakathil and $\mathrm{Kirk}^{4}$ were.

\section{Procedure}

Lines were presented in four different spatial conditions: horizontal, vertical, radial-down, and radial-up (Figure 2). Horizontal lines were presented flat on a tabletop in the transverse plane with the midpoint of the line $30 \mathrm{~cm}$ from the

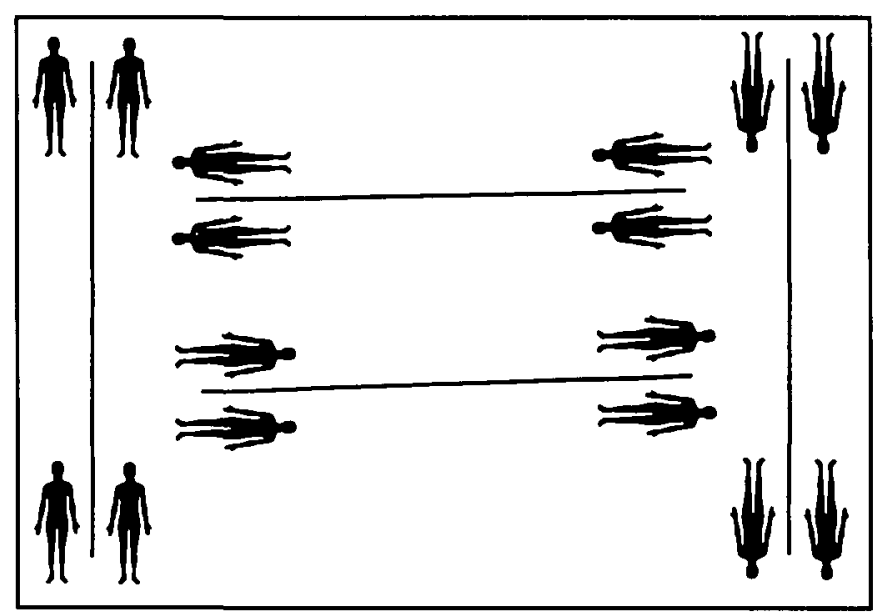

Figure 1: Labelling possibilities for lines in the four spatial orientations. The two inner lines demonstrate positioning of labels for horizontal lines. The two outer lines demonstrate label positioning for radial-up, radial-down, and vertical lines.

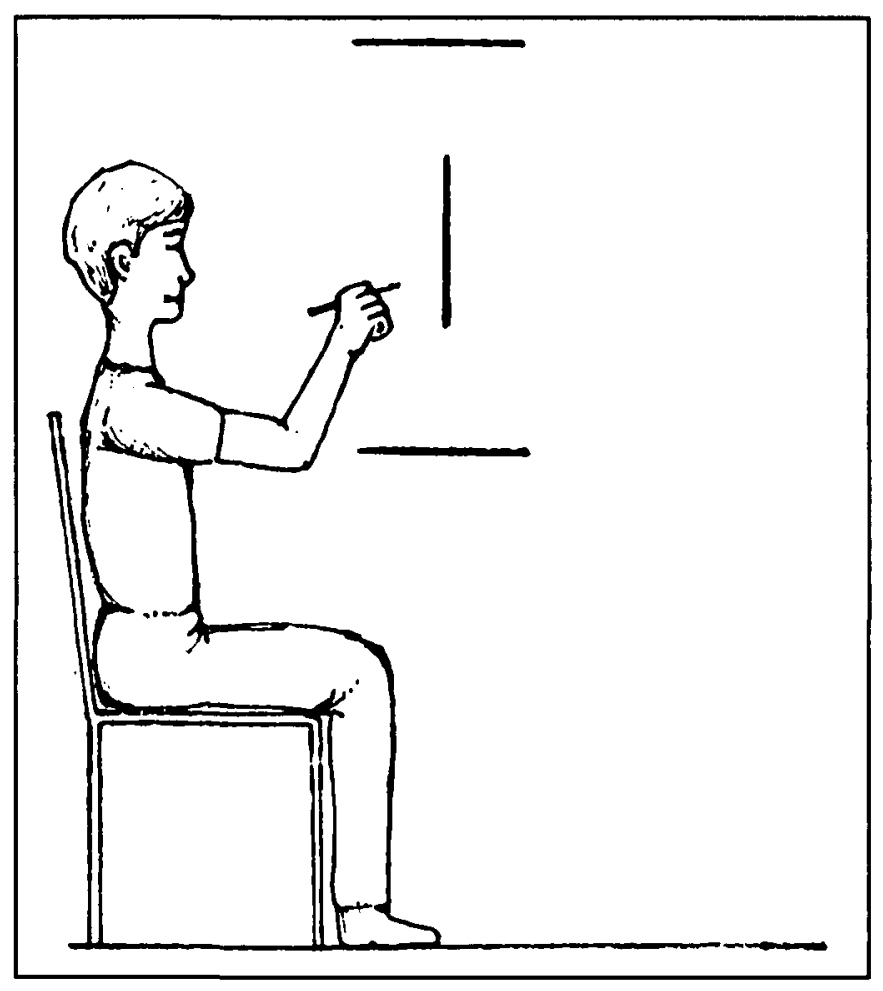

Figure 2: Location of stimuli in relation to subject. (Reprinted with permission from Neurology, 1994; 44: 703-706.4) 
subject's sternum in the body midline and with the line parallel to the coronal plane. Radial-down lines were also presented flat on a tabletop in the transverse plane and oriented along the midsagittal plane, with midpoint $30 \mathrm{~cm}$ from the body. Both horizontal and radial-down lines were presented $35 \mathrm{~cm}$ below eye level. Vertical lines were presented on a wall $30 \mathrm{~cm}$ from the subject, with the midpoint at the subject's eye level. Radial-up lines were presented in the transverse plane $35 \mathrm{~cm}$ above eye level, oriented along the midsagittal plane, with midpoints 30 $\mathrm{cm}$ from the body.

For each spatial condition, half of the lines were presented with the figures' heads pointing in one direction and the other half with heads pointing in the other direction. For example, in the vertical orientation, the figures were upright for half of the lines to be bisected and upside down for the other half. Each line length was presented twice in each combination of spatial condition and figure head position. Thus, with four spatial conditions, six line lengths, two figure head positions, there was a total of 96 lines for each subject to bisect. Stimuli were grouped according to spatial orientation and presented in a pseudorandomized fashion. Subjects were asked to use a pencil to bisect each line as accurately as possible. The duration of testing averaged about 45 minutes. Measurements of subjects' bisection errors were done manually after testing was completed. No feedback about performance was given to the subjects.

\section{Results}

Line bisection error (LBE) was measured to the nearest millimetre from the true midpoint. Deviations to the left of, below, or nearer than the true midpoint were assigned a negative value. Deviations to the right of, above, or further than true midpoint were assigned a positive value. As previously discussed, each spatial condition could have two different labelling possibilities: one with the figures' heads pointing in one direction, the other with the heads pointing in the other direction. For each spatial condition, data from each labelling possibility were pooled across line lengths and compared with data from the opposite labelling possibility using paired t-tests with the statistical program SPSS. Because multiple t-tests were performed, the Bonferroni method was used to correct $p$ values. Results are reported in the Table.

For every spatial condition, lines were misbisected with a significant bias towards the figures' heads, that is, towards the pictorially defined top of the line. Bisection of vertical lines with figures' heads pointed upward showed a mean LBE above true midpoint. When the figures' heads pointed downward, vertical lines were still misbisected above true midpoint, but the magnitude of the error was significantly reduced. In the radialdown condition, lines were misbisected distal to true midpoint. Again, the mean LBE was significantly reduced when the figures' heads pointed toward rather than away from the subject. Lines in the radial-up condition were misbisected significantly proximal to true midpoint. Again, the mean LBE was significantly different depending upon labelling situations with the mean LBE significantly more proximal when the figures' heads pointed toward the subject. Horizontal line bisection with figures' heads pointing left had a mean LBE left of the true midpoint. Bisection of horizontal lines with figures' heads pointing right also had a mean LBE left of true midpoint, but the mean
Table: Line bisection errors for groups of lines with opposing labels.

Line Group
$\begin{aligned} & \text { Mean Error } \\ & \text { (cm) }\end{aligned}$ $\begin{gathered}\text { SD } \\ \text { (cm) }\end{gathered}$

LBE was significantly reduced. Thus, in each spatial orientation, the mean LBE demonstrated a significant bias toward the figures' heads.

\section{Discussion}

The direction of mean LBE for all spatial conditions was the same as that reported in previous studies. ${ }^{1.3 .4}$ In each spatial condition, the magnitude of the mean LBE depended upon the orientation of the figures at either end of each line.

Control subjects misbisect unlabelled vertical lines above true midpoint. ${ }^{1.46} \mathrm{An}$ unlabelled vertical line has a frame of reference determined by the visual field. A misbisection above the true midpoint may demonstrate an upper visual field bias, a topward attentional bias, or both. Jeerakathil and $\mathrm{Kirk}^{4}$ suggested that the labels TOP and BOTTOM provide an alternative reference frame superimposed over the visual reference frame. ${ }^{4}$ In the present study, the mean LBE magnitude also depended upon the figures' position with the mean LBE significantly reduced when the figures' heads pointed down. An internal frame of reference appears to be adopted after viewing the labels at either end of the line. The mean $\mathrm{LBE}$ remains above true midpoint for both figure positions, suggesting that the external visual fieldbased bias is stronger than the internal representational bias as induced by the labels.

Unlabelled radial-down lines are misbisected distal to true midpoint by control subjects. ${ }^{1.3 .4}$ When the frame of reference is determined by the visual field, a misbisection distal to centre can be explained by a bias toward the upper visual field, or a topward attentional bias, or both. Jeerakathil and Kirk ${ }^{4}$ also demonstrated the influence of verbal cues TOP and BOTTOM on radial-down line bisection. The mean LBE reduced significantly when the label TOP appeared at the end of the line nearest the subject. In the present study, the mean LBE was significantly reduced when the figures' heads pointed toward the subject. Again, these results can be explained by the adoption of 
an internal reference frame. Misbisection of unlabelled radial-up lines by control subjects is proximal to true midpoint. ${ }^{3}$ The mean LBE has been demonstrated to be in the direction of the top of the visual field. ${ }^{3,4}$ Jeerakathil and Kirk ${ }^{4}$ placed TOP and BOTTOM labels at either end of each line, but, in this condition only, they did not find a significant representational bias. In the present study, a significant increase in mean LBE occurred when the figures' heads pointed toward the subject, demonstrating that a representational bias exists in the radial-up condition also. The lack of a significant bias in this condition in the previous study may relate to the smaller number of subjects in that study.

For an unlabelled horizontal line, control subjects visually misbisect to left of centre. ${ }^{7.8} \mathrm{~A}$ horizontal line labelled with TOP and BOTTOM is misbisected in the direction of the label TOP. ${ }^{4}$ In the present study, the mean LBE was left of true midpoint for both figure positions, but the mean LBE was significantly less when the figures' heads pointed to the right. The bias toward the internal representation of top, while still present, was not as strong with the pictorial labels as it was with the verbal labels. We are accustomed to seeing human figures lying on their sides. Therefore, a horizontally oriented figure may not give a strong indication of top and bottom whereas an unaccustomed upside down figure may. This is in contrast to the label TOP, which can only suggest one spatial position, as we seldom view words lying on their sides.

Another possible explanation of the results is the effect of mental rotation. Shepard and Metzler ${ }^{9}$ demonstrated that during the transformation of mental images through increasing angles of rotation, there is an approximately linear increase in the time required to generate responses to the images. Thus, the rotation of upside-down figures could be expected to require a longer time than the rotation of horizontal figures, allowing the representation of top to become more deeply "engraved" with the presentation of upside-down figures. This provides a possible explanation of why the vertical and radial-down bisections showed a much greater difference in mean LBE depending upon figure position than did the horizontal condition.

Horizontal line bisection may be the most effective test for examining the effect of an internal vertical frame of reference. The horizontal line should remove the upper visual field bias, leaving only the internal representational bias. If we consider the difference in mean LBEs for the two figure positions as a vector, then this vector points in the direction of the figures' heads with a magnitude of $0.8 \mathrm{~mm}$. This vector depicts the effect of an internal representational bias of top upon horizontal line bisection. Vectors for the vertical and radial-down bisections both have a magnitude of $1.8 \mathrm{~mm}$ in the direction of the figures' heads, suggesting that the vector maintains the same direction, but increases in magnitude in these orientations. As suggested earlier, this increase in vector magnitude could be due to subjects being accustomed to seeing a prone figure. If we extend the vector argument to the study of Jeerakathil and Kirk, ${ }^{4}$ the strength of the representational bias in bisecting horizontal lines could be represented as a vector of $2.0 \mathrm{~mm}$ with a direction towards the label TOP. Vertical and radial-down line bisections had vectors of 2.0 and $1.8 \mathrm{~mm}$ respectively, both directed towards the label TOP. The strong similarity of these values suggests that the vector in our study may indeed have been weakened with the prone figure in the horizontal bisection.

This study supports the hypothesis that at least two separable biases underlie normal subjects' errors during line bisection. Subjects demonstrate 1) a visual field bias towards the upper visual field, and 2) a bias toward an internal representation of top. These separate biases appear to superimpose and may be thought of as vectors. If such attentional biases exist in control subjects, then they may be important in neglect patients as well. ${ }^{5}$

\section{ACKNOWLEDGEMENTS}

Supported in part by grants from the University of Saskatchewan, College of Medicine, and by the Saskatchewan Health Services Utilization and Research Commission. We thank Cynthia Kirk for the illustration and our subjects for volunteering their time.

\section{REFERENCES}

1. Shelton PA, Bowers D, Heilman KM. Personal and vertical neglect. Brain 1990; 113: 191-205.

2. Mennemeier M, Wertman E, Heilman KM. Evidence for multidirectional attentional systems in humans. Brain 1992; 115: 37-50.

3. Geldmacher DS, Heilman KM. Differences in radial line bisection above and below eye level [abstract]. J Clin Exp Neuropsychol 1992; 14: 35.

4. Jeerakathil TJ, Kirk A. A representational vertical bias. Neurology 1994; 44: 703-706.

5. Kirk A, Boyle C. "Radial neglect" is caused by vertical neglect of an internal representation [abstract]. Can J Neurol Sci 1994; 21 : 519.

6. Heilman KM, Watson RT, Valenstein E. Neglect and related disorders. In: Heilman KM, Valenstein E, eds. Clinical Neuropsychology, 3rd ed. New York: Oxford University, 1993; 248-298.

7. Bradshaw JL, Nettleton NC, Nathan G, Wilson L. Bisecting rods and lines: effects of horizontal and vertical posture on left-side underestimation by normal subjects. Neuropsychologia 1985 ; 23: $421-425$.

8. Halligan PW, Marshall JC. Line bisection in visuo-spatial neglect: disproof of a conjecture. Cortex 1989; 25: 517-522.

9. Shepard RN, Metzler, J. Mental rotation of three-dimensional objects. Science; 171: 701-703. 